\title{
Successful combined procedure of HeartMate II left ventricular assist device implantation and minimally invasive transapical aortic valve replacement
}

\author{
Ares K. Menon, MD, ${ }^{\mathrm{a}}$ Guido Dohmen, MD, ${ }^{\mathrm{a}}$ Andreas H. Mahnken, MD, MBA, ${ }^{\mathrm{b}}$ and \\ Rüdiger Autschbach, MD, PhD, ${ }^{\mathrm{a}}$ Aachen, Germany
}

Left ventricular assist device (LVAD) implantation is an accepted treatment for patients with end-stage heart failure, either as a bridge to transplantation or destination therapy. ${ }^{1,2}$ The newer continuous-flow LVAD HeartMate II LVAD (Thoratec Corp, Pleasanton, Calif) represents the current generation of devices ${ }^{3}$ and received CE mark and Food and Drug Administration approval, resulting in approximately 3500 implantations in patients worldwide. Severe aortic valve regurgitation remains a strict contradiction to the use of LVADs. We present a combined treatment with implantation of a HeartMate II LVAD after successful transapical aortic valve replacement in a 51year-old patient with end-stage heart failure and severe aortic regurgitation.

Transapical transcatheter aortic valve implantation (TA-AVI) was introduced to clinical use by Walther and colleagues ${ }^{4}$ in 2007 , after the first implantation in humans by Cribier and colleagues ${ }^{5}$ in 2002. TA-AVI reflects an alternative operative strategy to treat aortic valve disease in patients with advanced age and severe comorbidities, who present a high risk for open surgery with cardiopulmonary bypass (CPB).

\section{CLINICAL SUMMARY}

After failed conservative medical treatment, a 51-yearold male patient with end-stage heart failure and combined aortic valve disease with severe regurgitation was admitted to the intensive care unit. Cardiogenic shock developed in the patient, and he was treated with inotropes and later with levosimendan. The patient's left ventricular ejection fraction was $15 \%$, and his aortic valve showed severe regurgitation and moderate stenosis with an orifice area of 1.2 $\mathrm{cm}^{2}$ and considerable calcification. The cardiac index was $1.7 \mathrm{~L} / \mathrm{m}^{2}$. Additional findings were hypertension and

\footnotetext{
From the Clinic for Thoracic and Cardiovascular Surgery ${ }^{\mathrm{a}}$ and Department for Radiology, ${ }^{\mathrm{b}}$ University RWTH Aachen, Aachen, Germany.

Disclosures: Andreas H. Mahnken reports lecture fees from Bayer-Schering Pharma.

Received for publication Dec 31, 2009; accepted for publication Nov 15, 2010; available ahead of print Jan 31, 2011.

Address for reprints: Ares K. Menon, MD, University Hospital, Thoracic and Cardiovascular Surgery, Pauwelsstrasse 30 D-52074, Aachen, Germany (E-mail: amenon@ukaachen.de).

J Thorac Cardiovasc Surg 2011;142:708-9

0022-5223/\$36.00

Copyright (C) 2011 by The American Association for Thoracic Surgery

doi:10.1016/j.jtcvs.2010.11.019
}

a positive test result for heparin-induced thrombocytopenia II antibodies, reflected by a severe decrease in thrombocytes. Therefore, the patient was treated with argatroban instead of heparin.

Because the patient's left ventricular function did not recover, an LVAD implantation was planned as a bridgeto-transplantation procedure. His aortic valve disease combined with severe regurgitation made LVAD implantation unfeasible. TA-AVI was planned to avoid a conventional aortic valve replacement. The latter would have caused additional damage not only to the left ventricle but also to the already impaired right ventricle.

In the first procedure, a 26-mm Edwards SAPIEN aortic valve (Edwards Lifesciences, Irvine, Calif) was successfully implanted under LVAD stand by a transapical approach through a left-sided anterolateral thoracotomy under rapid ventricular pacing, as described previously. ${ }^{4}$ The patient was hemodynamically stable and could be extubated within 2 hours after surgery. Postoperative transesophageal echocardiography showed only a mild paravalvular leak with no relevance. The mean valve gradient was $12 \mathrm{~mm} \mathrm{Hg}$.

Despite this promising course, the patient showed no recovery tendency in left ventricular function and could not be weaned from inotropes or levosimendan. Therefore, the second step was initiated.

LVAD implantation was performed through a median sternotomy, and CPB was begun under mild hypothermia. The Thoratec HeartMate II LVAD was implanted at the apex of the left ventricle under beating heart conditions, and the pump was positioned in a pre-peritoneal pocket. The Dacron outflow graft was anastomosed to the ascending aorta end to side, as recommended by the manufacturers. Because the patient's right ventricular function was severely impaired, inhalative nitric oxide was administered and milrinone was given intravenously, enabling weaning from CPB and closure of the chest. The preoperative application of clopidogrel, argatroban, and aspirin resulted in bleeding, and surgical reexploration was necessary 18 hours postoperatively.

Pulmonary function was stabilized after application of sildenafil, and the patient was successfully weaned from the ventilator after 4 days. Inotropes were discontinued after 6 days. Phenprocoumon was administered, and after 8 days the patient was transferred from the intensive care unit to an 


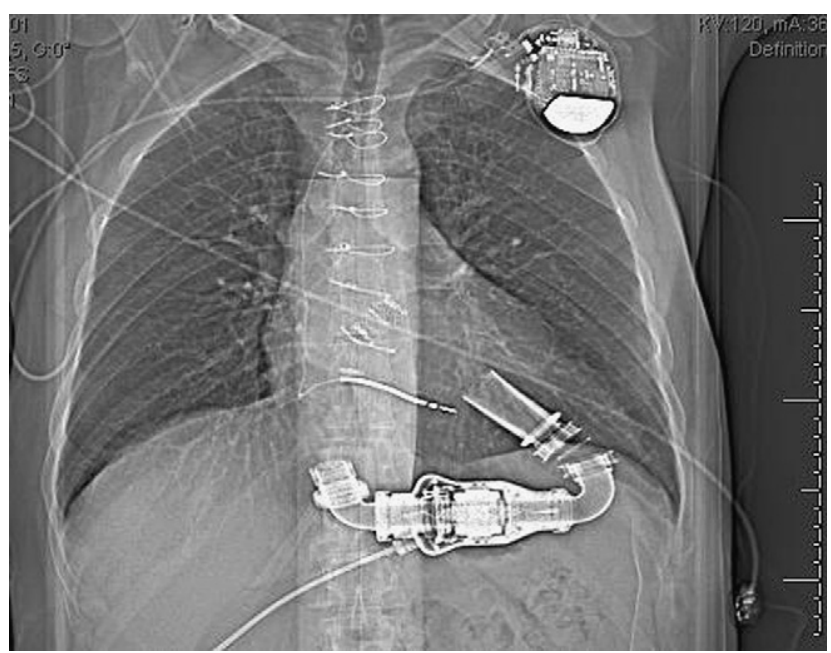

FIGURE 1. Overview of the computed tomography scan exhibiting the transapical aortic valve prosthesis and the LVAD.

intermediate care unit. Wound healing and mobilization were uneventful, and the patient was transferred to a rehabilitation facility after he was successfully trained in the use of all equipment.

Transthoracic echocardiography showed good performance of both the LVAD and the aortic valve prosthesis, although a mild paravalvular leakage was observed.

After further successful training in the CoaguCheck (Roche Diagnostics, Indianapolis, Ind) international normalized ratio self-testing equipment with a target area of 2.0 to 2.5 , the patient is now receiving out of hospital care and listed for heart transplantation. Ten weeks postoperatively, an implantable cardiodefibrillator was implanted because of reoccurrence of ventricular arrhythmias.

The actual transesophageal echocardiogram 12 months after implantation showed a good performance of the SAPIEN aortic valve and HeartMate II LVAD. The paravalvular leakage had completely disappeared, but a mild, central regurgitation jet was observed. The right ventricle remained impaired, but the size decreased, and the patient was classified with New York Heart Association class I.
Twelve months after discharge, the patient is able to manage the 6-minute-walk with $500 \mathrm{~m}$.

These clinical findings correlate well with a 64-multislice computed tomography scan (Figure 1) showing perfect LVAD position and unimpaired SAPIEN valve position.

To our knowledge, this is the first successful implantation of a permanent axial LVAD after transapical aortic valve replacement as a bridge to transplantation. The results show stable conditions, with no displacement or thrombosis of the aortic valve prosthesis. Even the mild paravalvular leak had completely disappeared compared with the initial findings, with no deterioration under phenprocoumon and high flow of the LVAD. The excellent clinical recovery of the patient has led to the listing for orthotopic heart transplant.

\section{CONCLUSIONS}

In cases of severe aortic valve disease, especially moderate to severe aortic regurgitation, which represents a strict contraindication for axial LVAD implantation, this combined approach with TA-AVI and LVAD implantation may be an appropriate solution for select patients with end-stage heart failure if there is a certain grade of calcification to fixate the new valve for a time-consuming, necessary open valve implantation. Further experience is needed to discern whether this combination therapy will be successful after long-term use, particularly in the increasing number of patients who are not transplantation candidates, the destination therapy group.

\section{References}

1. Frazier OH, Rose EA, Oz MC, et al. Multicentre clinical evaluation of the HeartMate vented left ventricular assist system in patients awaiting heart transplantation. J Thorac Cardiovasc Surg. 2001;122:1186-95.

2. Rose EA, Gelijns AC, Moskowitz AJ, et al. Long-term mechanical left ventricular assistance for end-stage heart failure. $N$ Engl J Med. 2001;345: 1435-43.

3. John R, Kamdar F, Liao K, et al. Improved survival and decreasing incidence of adverse events with the HeartMate II left ventricular assist device as bridge-totransplant therapy. Ann Thorac Surg. 2008;86:1227-35.

4. Walther T, Simon P, Dewney T, et al. Transapical minimally invasive aortic valve implantation. Circulation. 2007;116(11 Suppl):I240-5.

5. Cribier A, Eltchaninoff H, Bash A, et al. Percutaneous transcatheter implantation of an aortic valve prosthesis for calcific aortic stenosis: first human case description. Circulation. 2002;106:3006-8. 